C. Benedi, E. Rico, J. Guemes \& A. Herrero (Eds.) Flora Ibérica. Vol. XIII. CSIC. Madrid. pp. 38-40.

PRESTON, C.D. \& J.M. CROFT -1997-. Aquatic plants in Britain and Ireland. Hartley Books. Colchester.

ROEFOLS, J.G.M. -1983-. Impact of acidification and eutrophication on macrophyte communities in soft waters in the Netherlands. I. Field observations. Aquatic Botany 17: 139-155.

Dirección de los autores. Departamento de Biología Vegetal y Ecología. Facultad de Farmacia. Universidad de Sevilla. C/. Profesor García González s/n. 41012 Sevilla. *Autor para correspondencia: pgarcia@us.es

\title{
146. NARCISSUS CAVANILLESII EN LA CUENCA DEL GUADIANA: HACIA UNA CONSERVACIÓN TRANSFRONTERIZA
}

\author{
Isabel MARQUES ${ }^{1 *}$, Antonia ROSSELLÓ-GRAELL ${ }^{1} \&$ David DRAPER $^{2}$
}

Recibido el 22 de Octubre de 2008, aceptado para su publicación el 2 de enero 2009 Publicado "on line" en marzo de 2009

\section{Trans-boundary conservation of Narcissus cavanillesii in the Guadiana valley}

Palabras clave. Fragmentación de poblaciones, comportamiento metapoblacional, conservación transfronteriza, Portugal, España.

Key words. Fragmented populations, metapopulational behavior, trans-boundary conservation, Portugal, Spain.

Narcissus cavanillesii A. Barra \& G. López (Amaryllidaceae) es una especie endémica del sur de la Península Ibérica y norte de África. Se trata de un pequeño geófito de floración otoñal contemplado en los Anexos II y IV de la Directiva Hábitat 92/43/CEE y en el Anexo 2 de la Ley 42/2007, de 13 de diciembre, del Patrimonio Natural y de la Biodiversidad (bajo el nombre de $N$. humilis).

En la Península Ibérica, el área de distribución de $N$. cavanillesii se restringe al cuadrante sudoeste, estando referenciada para la provincia portuguesa de Alto Alentejo (Malato-Beliz, 1977; Rosselló-Graell et al., 2003a) e para las provincias españolas de Cádiz, Córdoba, Huelva, Sevilla (Valdés et al., 1987), Málaga (MA 22495) y Badajoz (Devesa, 1995; Rivas Goday \& Ladero Álvarez, 1973). La cita para la provincia de Cáceres no debe de ser considerada pues se trata de error de identificación, ya confirmado por Barra (2002). Aunque hasta el momento no se conoce la 
presencia de $N$. cavanillesii en el levante de la Península Ibérica, la ocurrencia del híbrido $N$. xperezlarae en la Comunidad Valenciana (Soler, 1998) supone su presencia en la zona al menos en el pasado.

$N$. cavanillesii se puede reproducir por autogamia pero la mayor producción de frutos y semillas procede de polinizaciones xenógamas (Rosselló-Graell et al., 2007) efectuadas por insectos generalistas, como Halictus sp. (Rosselló-Graell et al., 2003b) y las poblaciones pequeñas no son suficientemente atractivas para los polinizadores (Marques et al., 2007). Además, las barreras reproductivas de $N$. cavanillesii son muy débiles y la existencia de hibridación es frecuente (Marques et al., 2007). Por todo estos factores las poblaciones pequeñas de esta planta presentan un riesgo elevado de desaparición. De hecho, la ocurrencia en la Comunidad Valenciana de poblaciones aisladas de $N$. xperezlarae sugiere el desplazamiento de $N$. cavanillesii por el híbrido (Marques et al., 2007).

En España, las principales poblaciones conocidas se localizan hasta los 1000 m.s.n.m. en prados abiertos no arados. Varias poblaciones han encontrado refugio en márgenes de carreteras que por el tipo de gestión que tienen estos espacios permiten su existencia. $\mathrm{Su}$ ocurrencia en zonas cercanas a vías de comunicación supone una consecuente amenaza para su conservación futura en el caso de que se realicen obras en las citadas infraestructuras. Un ejemplo reciente de esta situación es el ensanchamiento de la carretera de Medina Sidonia a Chiclana de la Frontera (carretera A-390 en Cádiz) donde se ha podido constatar la destrucción de más del $80 \%$ de la población de $N$. cavanillesii (obras de acondicionamiento de dicha carretera realizadas en 2006) así como de las poblaciones de $N$. xperezlarae, $N$. serotinus, $N$. viridiflorus, y $N$. xalleniae, correspondiendo además esta localidad al locus typicus de este último taxón (DonnisonMorgan, 2000). A pesar de esta situación de amenaza, este taxón no se encuentra en el Catálogo Andaluz de Especies Amenazadas (Devesa \& Ortega, 2004) ni en la Lista Roja de la Flora Vascular de Andalucía (Cabezudo et al., 2005). No obstante, N. cavanillesii se encuentra registrado en el Catálogo Regional de Especies Amenazadas de Extremadura con la clasificación "De interés especial" del Decreto 37/2001 de 6 Marzo, D.O.E. número 30 (Devesa \& Ortega, 2004). Esta designación exige la existencia de un Plan de Manejo que determine las medidas para mantener las poblaciones en unos niveles adecuados (D.O.E. número 30; Devesa \& Ortega, 2004), aunque no se definen tales niveles.

En Portugal se alertó del peligro de extinción de la especie hace casi dos décadas (ICN, 1990). Como medida de conservación se incluyó el área donde se ubicaba la única población conocida en ese momento (MalatoBeliz, 1977) en la Red Natura 2000 como un Lugar de Interés Comunitario, "Sitio Guadiana/Juromenha (Resolução do Conselho de Ministros n¹42/97 de 28 de agosto)". Posteriormente se localizó la segunda población portuguesa de N. cavanillesii (Rosselló-Graell et al., 2003a). Ambas poblaciones se encuentran en la cuenca del río Guadiana constituyendo el límite occidental de la distribución del taxón. En Portugal, N. cavanillesii se cataloga como una especie "Criticamente Amenazada" (CR) B2ab(ii, iv) de acuerdo con los criterios de la IUCN (2001) por su pequeña área de ocupación, elevada fragmentación y continua disminución en su área de ocupación y en el número de poblaciones (Rosselló-Graell et al., 2004). Debido al impacto de la construcción del embalse del Alqueva en el río Guadiana, las dos poblaciones portuguesas de $N$. cavanillesii han sido monitorizadas desde 2001 para comprender su dinámica poblacional así como identificar sus condicionantes bióticos y abióticos. La información obtenida permitirá formular acciones de compensación para la conservación de esta especie en Portugal. En 
el ámbito de los estudios de monitorización que se han realizado se localizaron dos nuevas poblaciones de $N$. cavanillesii, uno en cada orilla del río Guadiana:

Narcissus cavanillesii A. Barra \& G. López in Anales Jard. Bot. Madrid 41(1): 202 (1984).

ESPAÑA, BADAJOZ: Olivenza, Puente de Ayuda. 29SPC5993, 155 m, margem esquerda do Rio Guadiana, perto da antiga ponte, com Quercus rotundifolia, 22-X-2001, Antónia Rosselló-Graell, s.n. (LISU 225068).

PORTUGAL, ALTO ALENTEJO: Elvas, Ajuda. 29SPC5793, $127 \mathrm{~m}$, margem direita do rio Guadiana a Sul da nova Ponte da Ajuda, perto da Herdade de S. Rafael, junto a afloramentos de xisto, com Urginea maritima e Scilla autumnalis, 15-X-2007, Isabel Marques, MARQ1001. (LISU 225069).

La primera población de $2-3 \mathrm{~m}^{2}$ se localizó en septiembre de 2001 y a lo largo de los años ha presentado un crecimiento fluctuante, con 46 individuos reproductores censados en 2001, 76 en 2002, 87 en 2003, 25 en 2004, 145 en 2005, 656 en 2006 y 84 en 2007. La segunda población, de pequeñas dimensiones $(30 \mathrm{x}$ $40 \mathrm{~cm})$ y con 38 individuos reproductores, se encontró en octubre de 2007.

Además de la población principal de $N$. cavanillesii (Malato-Beliz, 1977), existen diversas poblaciones de reducido tamaño en el margen portugués del río Guadiana. Estos grupos formados por entre 4 y 30 individuos reproductores presentan una asincronía en su floración y una dinámica poblacional fluctuante basado en los censos efectuados anualmente entre 2001 y 2007. Este comportamiento es también característico de la otra población portuguesa situada en Montes Juntos (LISU 173700; Rosselló-Graell et al., 2003a) cuyos núcleos presentan distintos patrones reproductivos (Rosselló-Graell et al., submitted).

Otras poblaciones citadas históricamente para otros municipios cercanos no pudieron localizarse durante las prospecciones de campo efectuadas en el marco de este trabajo. Este el caso de las poblaciones situadas en el Campus de la Facultad de Ciencias de Badajoz (UNEX10345) ya que probablemente hayan desaparecido a causa de las alteraciones que ha experimentado el campus (J. López Martínez, comunicación personal). Tampoco se observaron las poblaciones citadas para la finca de Casablanca, también en la provincia de Badajoz (UNEX18933), a pesar de las búsquedas realizadas durante varios años. No obstante, en octubre de 2005, A. Sánchez localizó nuevas poblaciones de $N$. cavanillesii cerca del embalse de Mirandilla (A. Sánchez, comunicación personal).

La existencia de pequeñas poblaciones, que no son constantes demográficamente a lo largo de los años es una característica frecuente en $N$. cavanillesii y la existencia confirmada de flujo genético entre estas poblaciones (I. Marques, datos inéditos) sugiere un comportamiento metapopulacional en esta planta. Considerando la posibilidad de metapoblaciones, la persistencia de $N$. cavanillesii dependería de la red de poblaciones existente (Tremblay et al., 2006). En este sentido, las estrategias de conservación de $N$. cavanillesii, en lugar de centrarse en poblaciones concretas deberían englobar la red de poblaciones ya que aunque estas sean pequeñas juegan un papel importante en las relaciones de flujo genético. El valor de esas pequeñas poblaciones es aún mayor cuando esta especie presenta marcadas oscilaciones de efectivos reproductores a lo largo de los años. Una vez que tanto las poblaciones de Badajoz (España) y del Alentejo (Portugal) son muy escasas y fragmentadas pero se encuentran relativamente próximas, la conservación de esta especie debería ser abordada de forma transfronteriza considerando las poblaciones situadas a ambos lados de la frontera LusoEspañola. 
AGRADECIMIENTOS. Proyecto promovido por EDIA, S. A. y cofinanciado por EDIA, S. A. y por FEDER. Los autores agradecen la información cedida por Josefa López Martínez y Angel Sánchez.

\section{BIBLIOGRAFÍA}

BARRA, A. -2002- Notas sobre Narcissus L. (Amaryllidaceae), II. Anales Jard. Bot. Madrid 59(2): 351.

CABEZUDO, B., S. TALAVERA, G. BLANCA, C. SALAZAR, M. CUETO, B. VALDÉS, J. E. HERNÁNDEZ BERMEJO, C. M. HERRERA, C. RODRÍGUEZ HIRALDO y D. NAVAS. -2005- Lista Roja de la Flora Vascular de Andalucía. Consejería Medio Ambiente. Sevilla, España. 126 pp.

DEVESA, J. -1995-Vegetación y Flora de Extremadura: 638. Universitas Editorial, Badajoz.

DEVESA, J.A y A. ORTEGA. -2004- Especies vegetales protegidas en España: plantas vasculares. Ministerio de Medio Ambiente. Organismo Autónomo Parques Nacionales.

DONNISON-MORGAN, D. -2000- A newly described wild hybrid in Narcissus. RHS. New plantsman 7: 41-42.

ICN. -1990- Lista de espécies a proteger em Portugal Continental. Lisboa. Portugal.

IUCN. -2001- The IUCN red list of threatened species 2001 categories and criteria, V 3.1. Gland.

MALATO-BELIZ, J. -1977- Braxireon humile (Cav.) Rafin-Nouvelle Amaryllidacée pour le Portugal. Candollea 32: 249-254.

MARQUES, I., A. ROSSELLÓ-GRAELL, D. DRAPER \& J. M. IRIONDO. -2007-Pollination patterns limit hybridization between two sympatric species of Narcissus (Amaryllidaceae). Am. J. Bot. 94(8) :1352-1359.

TREMBLAY, R., E. MELÉNDEZ-ACKERMAN \& D. KAPAN. -2006-Do epiphytic orchids behave as metapopulations? Evidence from colonization, extinction rates and asynchronous population dynamics. Biol. Conserv. 129: 70-81.

RIVAS GODAY, S. y M. LADERO ÁLVAREZ -1973- Nuevas aportaciones a la flora pacense. Anales Real Acad. Farm. 3: 283.

ROSSELLÓ-GRAELL, A., I. MARQUES y D.
DRAPER. -2003a- Segunda localidad de Narcissus cavanillesii A. Barra \& G. López (Amaryllidaceae) para Portugal. Acta Bot. Malacitana 28:196-197.

ROSSELlO-GRAELL, A., E. SALVADO, S. ALBANO, D. DRAPER y A.I.D. CORREIA. -2003b-Conservation programme for Narcissus cavanillesii (Amaryllidaceae) in Portugal. Bocconea 16(2) : 853-856.

ROSSELLO-GRAELL, A., D. DRAPER, I. MARQUES \& J. M. IRIONDO. -2004Conservation of threatened Narcissus cavanillesii A. Barra \& G. López in Portugal. http://www. nerium.net/plantaeuropa/Download/Procedings/ Rossello.pdf

ROSSELLÓ-GRAELL, A., I. MARQUES, D. DRAPER \& J. M. IRIONDO. -2007- The role of breeding system in the reproductive success of Narcissus cavanillesii A. Barra \& G. López (Amaryllidaceae). Bocconea 21: 359-365.

ROSSELLÓ-GRAELL, A., I. MARQUES, D. DRAPER \& J. M. IRIONDO. -SubmittedFlowering and fruit set in a fragmented translocated population. Bocconea.

VALDÉS, B., S. TALAVERA y E. FERNÁNDEZGALIANO -1987- Flora Vascular de Andalucía Occidental Vol. III: 472. Ketres Editora S. A., Barcelona.

SOLER, J. X. -1998- Descubrimiento de Narcissus perezlarae Font Quer (Amaryllidaceae) en el levante español. Anales Jard. Bot. Madrid 56: 165-166.

${ }^{1}$ Museu Nacional de História Natural. Jardim Botânico. Rua da Escola Politécnica 58. 1250-102 Lisboa. Portugal. ${ }^{2}$ Depto. Biología Vegetal. E.T.S.I Agrónomos, Universidad Politécnica de Madrid. Av. Complutense s/n 28040 Madrid, España.

*Autora para correspondencia: icmarques@fc.ul.pt 